\title{
Using Allan Variance Based Semi-Parameter Model to Calibrate Pointing Errors of Alt-az Telescopes
}

\author{
Lingjie Yan ${ }^{1,2,3}\left(\mathbb{D}\right.$, Yongmei Huang ${ }^{1,2, *}$ and Yahui Zhang ${ }^{1,2}$ \\ 1 Key Laboratory of Optical Engineering, Chinese Academy of Sciences, Chengdu 610209, China; \\ jeyelche@163.com (L.Y.); zharvey@ioe.ac.cn (Y.Z.) \\ 2 Institute of Optics and Electronics, Chinese Academy of Science, Chengdu 610209, China \\ 3 College of Materials Science and Opto-Electronic Technology, University of Chinese Academy of Sciences, \\ Beijing 100039, China \\ * Correspondence: huangym@ioe.ac.cn; Tel.: +86-138-0819-9476
}

Received: 15 February 2018; Accepted: 10 April 2018; Published: 13 April 2018

check for updates

\begin{abstract}
In this paper, a new model called Allan variance based semi-parameter model is put forward to improve the pointing precision for alt-az telescopes. As for electro-optical tracking systems, the precision of line-of-sight (LOS) pointing is seriously influenced by both geometric and nonlinear error sources. In modeling part of the parameter model, a simplified mount model is set up to demonstrate impacts of geometric errors that are caused by imprecision during mechanical processes on LOS pointing, according to values of encoders and miss distances in charge-coupled-device (CCD) cameras. Considering the limitations of mount models, an improved hybrid model called the Allan variance based semi-parameter model is introduced to compensate for residual nonlinear errors in the calibration portion. Compared with the classical mount model, the Allan variance based semi-parameter model removes both geometric errors and nonlinear errors, and the LOS pointing precision has been remarkably improved after calibration of the proposed model.
\end{abstract}

Keywords: alt-az telescopes; LOS; pointing errors; nonlinear error; mount model; Allan variance; Allan variance based semi-parameter model

\section{Introduction}

Alt-az telescopes and analogous electro-optical systems are widely utilized for scientific and commercial applications such as optical communication, aerial photography and antenna stabilization. Control systems of telescopes require high-performance pointing precision to achieve accurate target acquisition; good reproducibility and less jitter are also essential. However, LOS pointing precision required for telescopes is far beyond the capacity of most manufacture and assembly processes. Owing to impacts of diverse error sources, actual LOS pointing directions of alt-az telescopes are not consistent with ideal directions. For the sake of reducing mechanical costs and improving the LOS pointing precision, modeling and calibration techniques should be studied in detail.

Various calibration methods were studied by many scholars to estimate and compensate for errors of alt-az telescopes in mechanical processes, which can be roughly divided into two categories. The first one is based upon numerical analysis of LOS pointing angles, such as the Spherical Function and extended interpolation model [1,2]. Nevertheless, it is not easy to attribute such models to physical error sources. Another method is based upon the kinematic analysis of error sources during mechanical processes, which establishes a kinematic mount model by relating pointing errors to axis positions.

Error sources of precision machines consist of geometric errors and nonlinear errors. Repeatable geometric errors account for $40-70 \%$ of the total errors in previous studies, and can be eliminated easily. However, remaining nonlinear errors are difficult to remove. Fisk [3] studied the pointing system 
of gimbaled inertial stabilized platforms. A physical mathematical model is employed to address geometric errors including misalignment of base, run-out of gimbal bearings, non-orthogonality of gimbal axes and so on. Keitzer [4] added arbitrary polynomial and harmonic terms to the classical mount model to expand the analytical form of geometric errors. Nevertheless, residual nonlinear errors cannot be removed by the added terms. To improve the pointing performances of physical kinematic model, a new hybrid model is established to compensate for both geometric errors and nonlinear errors of LOS pointing completely.

Offline modeling and online calibration processes of the proposed improved model were implemented to achieve better LOS pointing accuracy. First, a physical model based on comprehensive analysis of error sources was set up to demonstrate impacts of geometric errors on LOS pointing. Then, an improved hybrid model called Allan Variance based Semi-Parametric model (AVSPM) was proposed to compensate for residual nonlinear errors. The experiment proved that AVSPM significantly improved the LOS pointing precision of alt-az telescopes.

\section{Error Analysis and Modeling of Pointing Errors}

A typical alt-az telescope consists of optical imaging system (OIS) and target tracking system (TTS). In OIS, optical imaging sensors, such as CCD camera, infrared camera and visible spectrum camera, collect image information about targets of interest. In TTS, the two degrees-of-freedom servo system, rotating in azimuth axis and elevation axis, ultimately determines the LOS pointing performances of the instrument in three aspects: tracking to keep targets of interest within the field of view of CCD camera, stabilizing the LOS to obtain high-quality images, and determining the exact location according to position sensors. Some auxiliary equipment is also required in most alt-az telescopes, including gyroscopes, motors and encoders. The Denavit-Hartenberg (D-H) transformation [5] is adopted to demonstrate the mount model (MM). The objective of telescope rack modeling is to identify the geometric errors at specific positions and apply them to remove errors in practice.

In an ideal condition, the azimuth axis, the elevation axis and the optical axis are three perpendicular axes in zero elevation position. However, during the assembly and operation processes, perpendicularity errors, rotation errors due to variable relative motions, measurement errors owing to the offset and imprecision of encoders occur and influence each other. Most servo-control systems of telescopes employ the structure of inner stabilization loop and outer tracking loop. The former eliminates turbulences and movements of the rack and the latter guarantees that the LOS maintains pointing to targets of interest. As a result of drift, coulomb friction, backlash and offsets of auxiliary equipment, servo-control errors including stability and tracking errors, the final LOS pointing direction of the telescope is shaped by modeling the errors according to the order they occur. Besides the above-mentioned errors, nonlinear errors remain because of the influence of environmental factors such as wind, vibration, gravity, temperature and atmosphere [6,7], which cannot be demonstrated by a physical mount model but a statistical method.

The static pointing precision of telescope LOS pointing can be illustrated according to the inconsistency between the actual and ideal pointing direction. It is calculated by the value of encoders and miss distances in CCD camera in practice. As shown in Figure 1 [7], the error vector of LOS pointing is $\Delta=[\Delta A, \Delta E]^{T}$. The pointing errors can be expressed by the following formula:

$$
\left\{\begin{array}{c}
\Delta A=\hat{A}-A=f(A, E)+\varepsilon \\
\Delta E=\hat{E}-E=g(A, E)+\eta
\end{array}\right.
$$

where $\triangle A$ and $\Delta E$ are pointing errors in azimuth axis and in elevation axis, $\hat{A}$ and $\hat{E}$ are measurements of encoders in azimuth axis and in elevation axis, $A$ and $E$ are the actual angular positions, $f(A, E)$ and $g(A, E)$ are expressions of the mount model in azimuth axis and in elevation axis associated with both azimuth and elevation angles, and $\varepsilon$ and $\eta$ are independent errors related to measurements in azimuth axis and in elevation axis. 


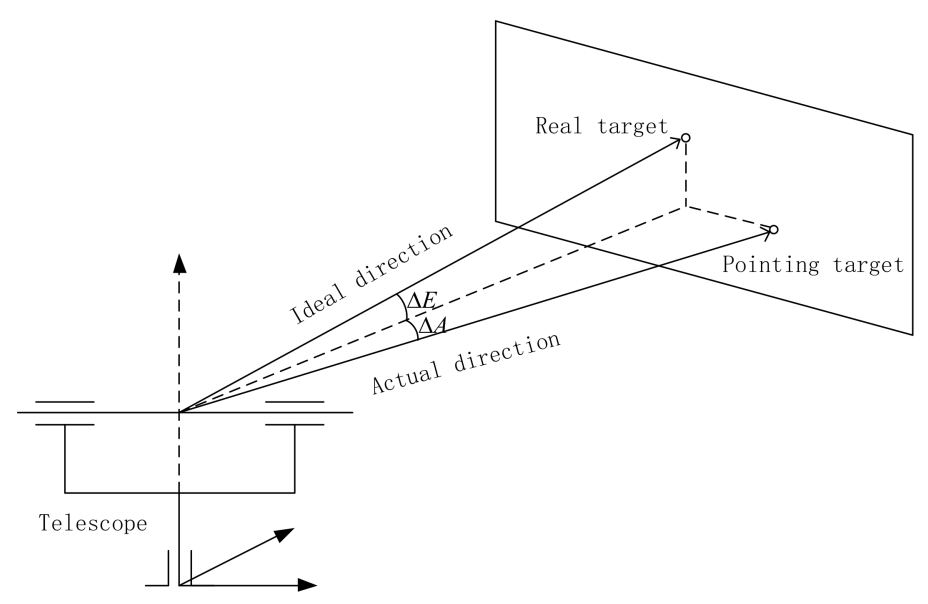

Figure 1. Pointing errors for telescopes.

\section{Online Calibration of Pointing Errors}

\subsection{Calibration of Geometric Errors}

With D-H convention, various error sources make corresponding impacts on the final LOS pointing. While the exact formula is too complicated to expand, a reasonable approximation is necessary. On account of the fact that each item of the geometric errors exerts a small rotation on telescope LOS vector, small-angle approximations [8] are considered suitable for the error angles, namely:

Rule 1: Each small rotation angle $\psi$ caused by geometric errors is considered to be an infinitesimal, namely $\sin (\psi)=\psi$, and $\cos (\psi)=1$.

Rule 2: Ignore high order error items, namely $\psi \cdot \psi=0$, where $\psi$ is the angle in radian.

After applying all rotations of error sources, the actual LOS is determined. The actual azimuth and elevation angle are $A+\Delta A$ and $E+\Delta E$, respectively. According to the small-angle approximation, the ultimate pointing error can be given as:

$$
\Delta=R^{T} x+v
$$

where $v=\left[\begin{array}{ll}\varepsilon & \eta\end{array}\right]^{T}$ indicates the vector of measurement related noises. The transformation matrix $R$ is denoted as:

$$
R=\left[\begin{array}{ll}
f(A, E) & g(A, E)
\end{array}\right]=\left[\begin{array}{cc}
1 & 0 \\
0 & 1 \\
1 & 0 \\
0 & 1 \\
-\sec E & 0 \\
\tan E & 0 \\
\sin 2 E \tan E & 0 \\
\cos 2 E \tan E & 0 \\
\cos 2 A \tan E \sin A & \cos A \cos 2 A \\
\sin 2 A \tan E \sin A & \sin 2 A \cos A \\
\cos 2 A \tan E \sin A & -\sin A \cos 2 A \\
\sin 2 A \tan E \cos A & -\sin A \sin 2 A \\
\tan E \sin A & \cos A \\
\tan E \cos A & -\sin A \\
0 & -\cot E
\end{array}\right]
$$


and $x$ is the parameter vector to be obtained.

\subsection{Estimation of Nonlinear Errors}

Imprecision during the manufacture, assembly, installation and operation processes has complex impacts on LOS pointing precision of alt-az telescopes. Thus, the mount model above-mentioned is unable to take all the cases into consideration, especially the highly coupled errors and environmental factors [9]. Error sources excluded are usually nonlinear and unavoidable, having complex statistical properties, and cannot be expressed exactly through kinematic modeling. Furthermore, small-angle approximations and errors related to measurements have increased the nonlinearity of residual errors.

To solve abovementioned problems and achieve higher pointing precision, a hybrid model that can handle with both geometric errors and nonlinear terms is proposed, called Allan variance based Semi-Parametric model.

An additional correction term for nonlinear errors is added to the mount model, thus Equation (2) can be rewritten as:

$$
\begin{gathered}
\Delta=R^{T} x+C+\mu \\
C=\left[\begin{array}{l}
\varepsilon_{c} \\
\eta_{c}
\end{array}\right]
\end{gathered}
$$

where $\Delta, R$, and $x$ are analogous to parameters of the mount model and $\mu$ is assumed to be a white noise. Equation (4) represents the AVSPM, which inherits parametric parts $R x$ from the mount model and adds the Allan variance correction item $C$ for residual nonlinear errors that are not considered in the mount model. Therefore, the AVSPM maintains high interpretability of geometric errors as mount models and retains certain flexibility of the nonlinear errors using a numerical method. AVSPM has been proven to outperform the mount model significantly in handling with complicated situations in practice.

Allan variance [10] method is a general method for analyzing frequency stability in time domain. This method is used to detect the contributions of all kinds of error sources in the noise data, and separate out and quantify these error sources. It is applicable to the analysis of the error characteristics of inertial sensors such as the fiber optic gyroscope and any other precision measuring instruments.

The Allan variance method is as follows: To sample the residual of mount model at sampling period $\tau_{0}$, total up to $N$ points. Divide all the points into $K$ groups, each group consists of $M$ sampling values $(M \leq(N-1) / 2)$. The sampling time for each group is $\tau=M \tau_{0}$, namely correlation time. The mean of each group is:

$$
\bar{w}_{k}(M)=\frac{1}{M} \sum_{i=1}^{M} w_{((k-1) M+i)}(k=1,2, \cdots, K)
$$

The Allan variance is defined as:

$$
\sigma_{A}^{2}(\tau)=\frac{1}{2(k-1)} \sum_{k=1}^{K-1}\left(\bar{w}_{k+1}(M)-\bar{w}_{k}(M)\right)^{2}
$$

The Allan variance can be obtained according to different correlation time $\tau$.

The relationship between the Allan variance and the bilateral power spectral density of the noise in the sampled data is as follows:

$$
\sigma_{A}^{2}(\tau)=4 \int_{0}^{\infty} S_{w}(f) \frac{\sin ^{4}(\pi f z)}{(\pi f z)^{2}} d f
$$


Formula above shows that a filter, whose transfer function is $\sin ^{4}(\pi f z) /(\pi f z)^{2}$, makes the noise of the output proportional to the Allan variance. Therefore, the Allan variance can separate, identify and quantify all kinds of noise items in the data.

Due to the influence of system noises, environmental noises and other factors, random errors occur in the output signal of the LOS pointing, including quantization noise (Q), zero bias stability (B), angle random walk $(\mathrm{N})$, rate ramp $(\mathrm{R})$, angular rate random walk $(\mathrm{K})$, exponential correlation noise (E), sinusoidal noise (S) and so on. If noise terms are independent in statistical properties, the Allan variance can be expressed as the sum of squares of various noise terms. In the actual data test, the first five items have the greatest contribution to the total error, therefore the total Allan variance can be represented by the first five items by approximation.

$$
\begin{gathered}
\sigma_{A}^{2}(\tau)=\sigma_{Q}^{2}(\tau)+\sigma_{N}^{2}(\tau)+\sigma_{B}^{2}(\tau)+\sigma_{R}^{2}(\tau)+\sigma_{K}^{2}(\tau) \\
=3 Q^{2} / \tau^{2}+N^{2} / \tau+B^{2}(2 \ln 2 / \pi)+K^{2} \tau / 3+R^{2} \tau^{2} / 2
\end{gathered}
$$

As the value of variance is very small in general case, the calculation of its standard deviation can help improve the fitting precision, thus the formula can be approximately expressed as:

$$
\sigma_{A}(\tau)=\sum_{n=-2}^{2} A_{n} \tau^{n / 2}
$$

When modeling random errors, white noise can be used directly as the driving noise of the system without modeling. The quantization noise can be transformed into the equivalent white noise, thus no further modeling is needed. To compensate for colored noises, corresponding models need to be established. For the remaining three kinds of colored noises in the alt-az telescope, the modeling process is as follows: First, obtain the power spectral density function of each colored noise, then the transfer function of each colored noise can be calculated through spectral decomposition theorem, and, finally, the differential equation can be derived from the transfer function of each colored noise. By adding all differential expressions of colored noises, the final model is set up.

As for zero bias stability, the power spectral density function is:

$$
S_{B}(w)=B^{2} / w
$$

According to the spectral decomposition theorem, the power spectral density function and the transfer function of shaping filter satisfy the following relationship.

$$
S(w)=|G(j w)|^{2}
$$

Thus, the transfer function of irrational form is obtained:

$$
G_{B}(j w)=B / \sqrt{j w}
$$

If the power spectral density function is irrational, then the calculated transfer function will be irrational too. Then, a favorable approximation is needed for the sake of analytical convenience. The zero bias instability can be approximated by first order Gauss-Markov process [11].

$$
\hat{G}_{B}(j w)=\beta B /(\beta+j w)
$$

After the Fourier inverse transformation, the differential equation of the zero bias instability is obtained.

$$
\dot{d}_{B}(t)+\beta d_{B}(t)=\beta B u_{1}(t)
$$

where $u_{1}(t)$ is the unit Gauss white noise. 
As for rate random walk, the power spectral density is:

$$
S_{K}(w)=K^{2} / w^{2}
$$

The transfer function of the shaping filter is:

$$
G_{K}(j w)=K / j w
$$

After the Fourier inverse transformation, the differential equation for random walk is obtained.

$$
\dot{d}_{K}(t)=K u_{2}(t)
$$

where $u_{2}(t)$ is the unit gauss white noise.

As for rate ramp, the power spectral density is:

$$
G_{R}(w)=R^{2} / w^{3}
$$

The transfer function of the shaping filter is:

$$
G_{R}(j w)=R /(j w)^{1.5}
$$

The rate ramp error can be approximated by the second order Gauss-Markov process [11]. The approximate transfer function is:

$$
\hat{G}(j w)=R /\left(-w^{2}+j \sqrt{2} w_{0} w+w_{0}^{2}\right)
$$

After the inverse Fourier transform, the differential equation of rate ramp is obtained:

$$
\ddot{d}_{R}(t)+\sqrt{2} w_{0} \dot{d}_{R}(t)+w_{0}^{2} d_{R}(t)=R u_{3}(t)
$$

Allan variance method can be employed to identify the output of the mount model. After getting all the Allan variance coefficients of noises, taking five groups of independent Gauss white noise as driven noises, the output of the five equations are added to form the final model. Thus, the Allan variance correction part $C$ for the remaining nonlinear errors is obtained:

$$
C=Q u_{1}+N u_{2}+\frac{\beta B u_{3}}{s+\beta}+\frac{K u_{4}}{s}+\frac{R u_{5}}{s^{2}+\sqrt{2} w_{0} s+w_{0}^{2}}
$$

where $u_{1}, u_{2}, u_{3}, u_{4}, u_{5}$ are five independent Gauss white noises; $s$ is the differential operator; and $Q, N, B, K, R$ denote quantization noise, angle random walk, zero bias stability, angular rate random walk, rate ramp, respectively, whose values are determined by calculation result of Allan variance.

\section{Experiment and Results}

The experiment was conducted to compare performances between the proposed AVSPM and the classical mount model. The experiment was carried out through a $1 \mathrm{~m}$ alt-az telescope, as shown in Figure 2, which was designed with an angular movement ranging from $0^{\circ}$ to $360^{\circ}$ in azimuth and $0^{\circ}$ to $90^{\circ}$ in elevation. Actual LOS directions and corresponding pointing errors of stars were obtained from the alt-az telescope. The 176 stars in use were randomly distributed in the whole sky and uniformly selected from the FK5 Catalogue, as demonstrated in Figure 3. 


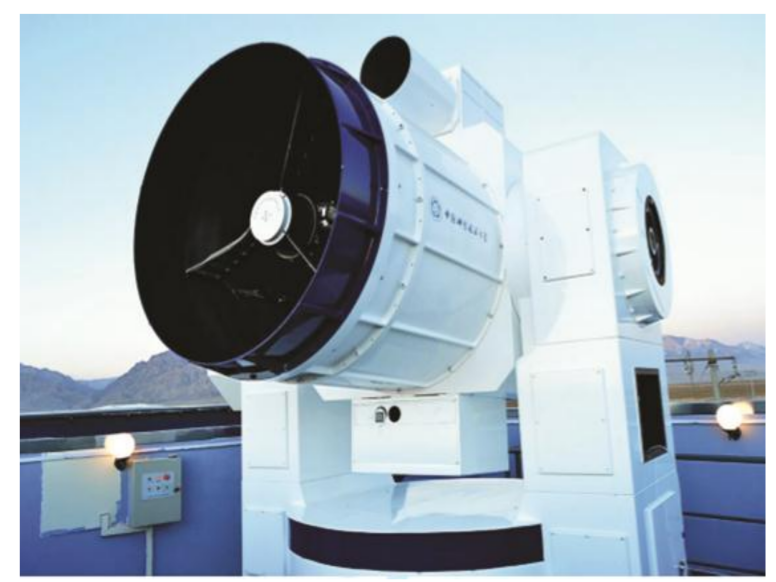

Figure 2. The alt-az telescope.

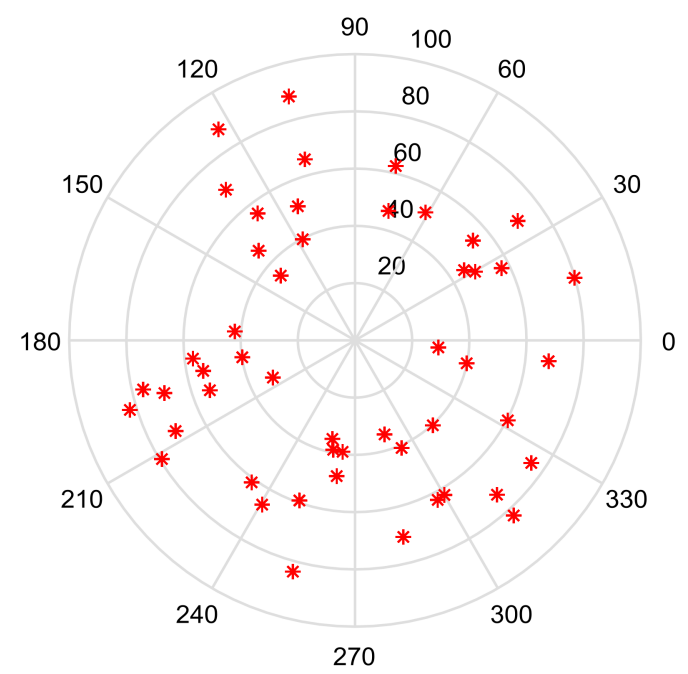

Figure 3. Star map distribution of the experiment.

The quantization noise, angle random walk, zero bias stability, rate ramp and angular rate random walk can be obtained by fitting, and the estimated values are:

$$
\begin{aligned}
& Q=A_{-2} / 3600 \sqrt{3} \\
& N=A_{-1} / 60 \\
& B=A_{0} / 0.6643 \\
& R=60 \sqrt{3} A_{1} \\
& K=3600 \sqrt{2} A_{2}
\end{aligned}
$$

As for zero bias stability, the relative amplitude error of the approximate transfer function and the real transfer function is:

$$
\varepsilon_{B}=10 * \log \left|\left(\left|\hat{G}_{B}(j w)\right|-\left|G_{B}(j w)\right|\right) /\right| G_{B}(j w)||=10 * \log \left|\sqrt{w} / \sqrt{1+w^{2}}-1\right|
$$

Considering low-frequency characteristics of the zero bias instability, the domain is restricted from $0.05 \mathrm{~Hz}$ to $0.1 \mathrm{~Hz}$; within this range, if $\beta$ equals $4 \mathrm{~Hz}$ and the amplitude error of the approximate 
transfer function $\hat{G}_{B}(j w)$ is less than $-3 \mathrm{~dB}$. Similarly, for rate ramp, the relative amplitude error of the approximate transfer function and the real transfer function is:

$$
\varepsilon_{R}=10 * \log \left|\left(\left|\hat{G}_{R}(j w)\right|-\left|G_{R}(j w)\right|\right) /\right| G_{R}(j w)||=10 * \log \left|\sqrt{w^{3}} / \sqrt{w^{4}+w_{0}^{4}}-1\right|
$$

Considering the low-frequency characteristics of the rate ramp, the domain is restricted from $0.005 \mathrm{~Hz}$ to $0.01 \mathrm{~Hz}$; within this range, if $w_{0}$ equals $0.05 \mathrm{rad} / \mathrm{s}$, the amplitude error of the approximate transfer function $\hat{G}_{R}(j w)$ is less than $-3 \mathrm{~dB}$.

The CCD camera must be calibrated before the experiment begins, aiming at determining the equivalent weight between miss distances and angles in azimuth and elevation axis. After that, the specific procedure to compare performances of the AVSPM and the classical mount model is as follows:

(1) Preparations for calibration process were made, namely blind-pointing measurements, including measuring LOS pointing errors at positions covering the whole sky and determining parameters of the mount model which most meet with the observed data.

(2) Active sampling such as blind-pointing measurements was carried out to acquire the correction coefficients of mount model. For the calibration portion, the residual errors after compensation of the mount model is necessary. The proposed AVSPM and conventional mount model were employed to calibrate LOS pointing errors at every pointing angle, making corrections available for the servo system and actuators. A numerical model called Spherical Harmonic Function Model (SHFM) was introduced for contrast. In SHFM, the spherical function is expanded into Fourier series, and then the zonal harmonic terms of series to fourth order are taken to shape the final model.

(3) While the LOS was turned to different directions to cover the whole sky within the movement range, all of the observed data were recorded synchronously, including pointing angles, corresponding errors and time.

As the baseline of these evaluation models, measurements of the original LOS pointing angles and corresponding errors are shown in Figure 4. Owing to the impacts of geometric and nonlinear errors, the LOS pointing was of poor pointing precision, 18.1477" in azimuth and 26.3599" in elevation, which is mainly caused by the inaccuracy during manufacture and assembly processes.

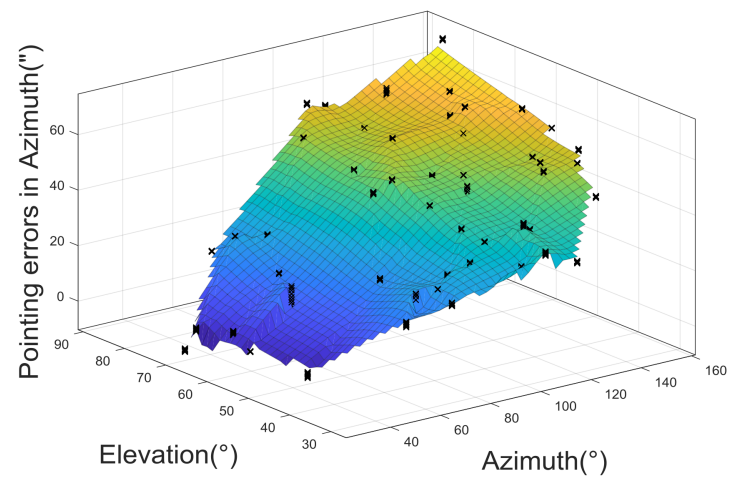

(a)

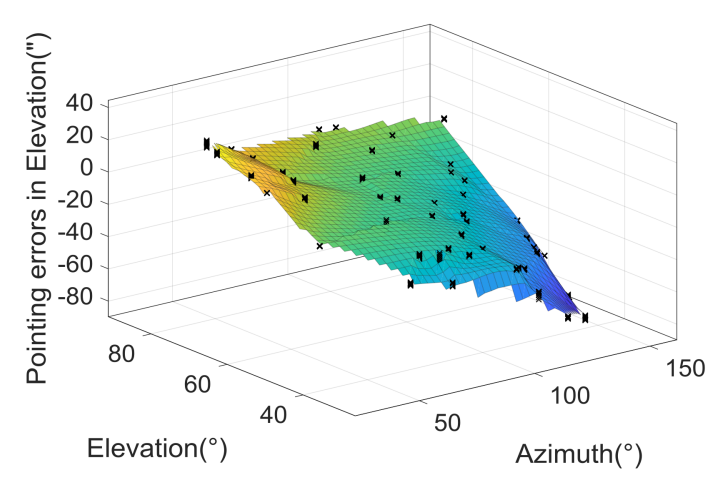

(b)

Figure 4. Original pointing errors of the alt-az telescope: (a) in azimuth; and (b) in elevation.

The mount model is then utilized to compensate for the geometric errors. Figure 5 and Table 1 present the remaining residual errors after compensation of mount model. It was shown that the mount model had remarkably compensated for geometric errors and improved the LOS pointing precision of alt-az telescopes. It was improved to $2.2376^{\prime \prime}$ in azimuth and $2.569^{\prime \prime}$ in elevation. The precision 
between mount model and spherical harmonic function model is similar. On the other hand, due to impacts of nonlinear factors excluded in the mount model, residual errors are not white noises.

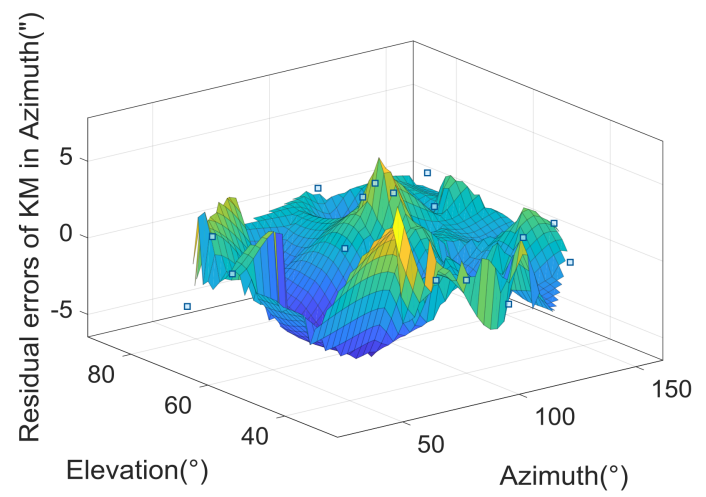

(a)

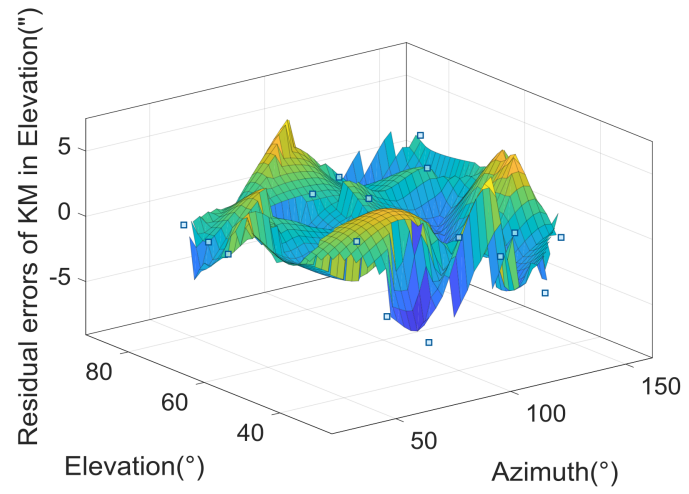

(b)

Figure 5. Residual errors and fitting surface of mount model: (a) in azimuth; and (b) in elevation.

Table 1. Pointing precision of the alt-az telescope after calibration.

\begin{tabular}{cccc}
\hline \multirow{2}{*}{ Pointing Model } & \multicolumn{3}{c}{ Pointing Precision (RMSE) of 1 m Alt-az Telescope } \\
\cline { 2 - 4 } & $\boldsymbol{\Delta} \boldsymbol{A}$ & $\boldsymbol{\Delta} \boldsymbol{~}$ & $\boldsymbol{\Delta}$ \\
\hline OE & $18.1477^{\prime \prime}$ & $26.3599^{\prime \prime}$ & $20.6719^{\prime \prime}$ \\
SHFM & $2.3655^{\prime \prime}$ & $2.7226^{\prime \prime}$ & $1.7112^{\prime \prime}$ \\
MM & $2.2376^{\prime \prime}$ & $2.569^{\prime \prime}$ & $1.6923^{\prime \prime}$ \\
AVSPM & $1.1261^{\prime \prime}$ & $1.3054^{\prime \prime}$ & $1.1542^{\prime \prime}$ \\
\hline
\end{tabular}

Consequently, the AVSPM is adopted to compensate for residual nonlinear errors. As shown in Figure 6 and Table 1, the result indicates that the AVSPM outperform the mount model remarkably in LOS pointing precision, namely 1.1261" in azimuth and 1.3054" in elevation. Furthermore, the residual errors of AVSPM are assessed according to the criterions provided by Eubank [12]. Because both geometric and nonlinear errors were removed by AVSPM, residual errors were determined to be white noises.

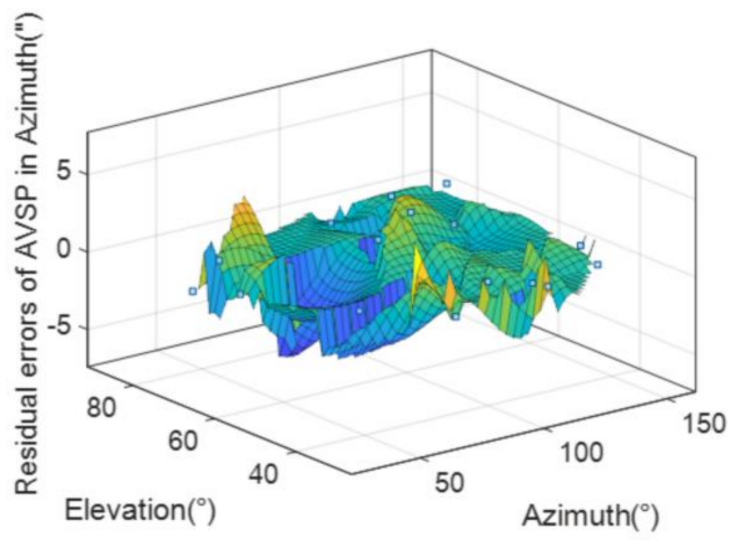

(a)

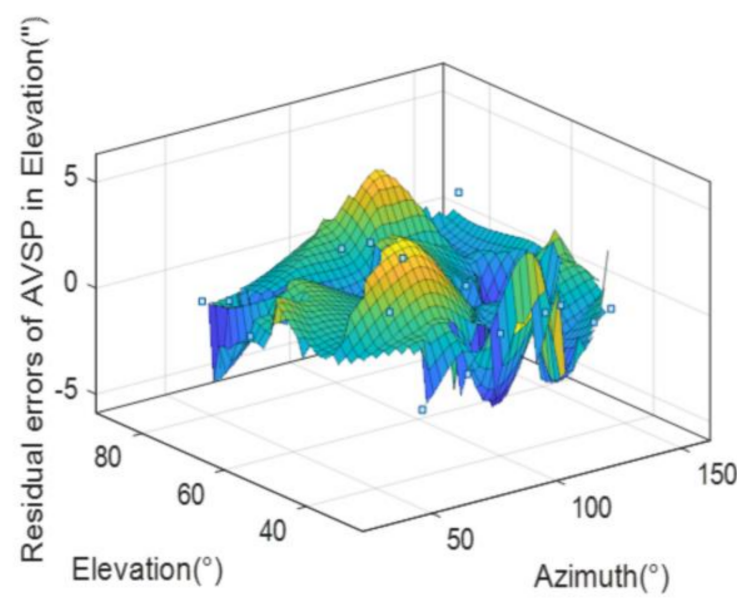

(b)

Figure 6. Residual errors and fitting surface of AVSPM: (a) in azimuth; and (b) in elevation.

It was proven that the LOS pointing precision of alt-az telescopes remarkably improved after calibration, and the proposed AVSPM achieved higher precision than the classical mount model. 


\section{Conclusions}

To improve the LOS pointing precision of alt-az telescopes, offline modeling and online calibration techniques are researched in this article from both theoretical and practical aspects.

A mount model was established to demonstrate and eliminate the impacts of geometric errors on the LOS pointing performance of alt-az telescopes, which was based on comprehensive analysis of geometric error sources and modeled with D-H transformation.

An improved model called the AVSPM was proposed to compensate for residual nonlinear errors excluded in the mount model. An experiment was carried out to evaluate properties of the proposed methods. As a result, the LOS pointing precision of telescope has been remarkably improved, and the AVSPM was proven to outperform the kinematic mount model. The AVSPM is also applicable to analogous two degrees-of-freedom equipment.

Acknowledgments: Comments and suggestions from T. Tang and Y. H. Luo during this research are very much appreciated.

Author Contributions: Lingjie Yan is the head of the research group that conducted this study. He contributed to the research with his original idea and he carried out the experiment and wrote this paper. Yahui Zhang participated in analyzing the theory and writing this paper. Yongmei Huang contributed to the research through her theoretical guidance and advice.

Conflicts of Interest: The authors declare no conflict of interest.

\section{References}

1. Zhang, X.X.; Wu, L.D. The basic parameters of telescope static point model. Acta Astromomica Sin. 2001, 42, 198-205.

2. Shi, H.L.; Yan, Y.H. Extended interpolation method and its applications in piecewise approximation. Comput. Appl. Math. 1992, 12, 229-236.

3. Fisk, J.W.; Rue, A.K. Confidence limits for the pointing error of gimbaled sensors. IEEE Trans. Aerosp. Electron. Syst. 2008, AES-2, 648-654. [CrossRef]

4. Keitzer, S.A.; Kimbrell, J.E.; Greenwald, D. Deterministic errors in pointing and tracking systems I: Identification and correction of static errors. In Proceedings of the International Society for Optics and Photonics Acquisition, Tracking, and Pointing V, Orlando, FL, USA, 1 August 1991; Volume 1482, pp. $415-424$.

5. Denavit, J.; Hartenberg, R.S. A kinematic notation for lower-pair mechanisms based on matrices. Trans. ASME J. Appl. Mech. 1955, 22, 215-221.

6. Sciavicco, L.; Siciliano, B. Modeling and Control of Robot Manipulators. Ind. Robot Int. J. 1996, 21, $99-100$.

7. Huang, L.; Ma, W.L.; Huang, J.L. Modeling and calibration of pointing errors with alt-az telescope. New Astron. 2016, 47, 105-110. [CrossRef]

8. Hong, H.J.; Zhou, X.Y.; Zhang, Z.Y.; Fan, D.P. Modeling and calibration of pointing errors using a semi-parametric regression method with applications in inertially stabilized platforms. Eng. Manuf. 2013, 227, 1492-1501. [CrossRef]

9. Mecozzi, A.; Antonelli, C.; Shtaif, M. Nonlinear propagation in multi-mode fibers in the strong coupling regime. Opt. Express 2012, 20, 11673-11678. [CrossRef] [PubMed]

10. Allan, D.W. Statistics of atomic frequency standards. Proc. IEEE 1966, 54, 221-230. [CrossRef]

11. IEEE Aerospace and Electronic Society. IEEE Standard Specification Format Guide and Test Procedure for Single-Axis Interferometric Fiber Optic Gyros; IEEE Press: Piscataway, NJ, USA, 1997.

12. Scimek, M.G. Smoothing and regression: Approaches, computation and application. J. R. Stat. Soc. 2003, $52,126-127$. 